\title{
Evaluation strategies for nuclear and radiological emergency and post-accident management
}

\author{
M. HIETE, V. BERTSCH, T. COMES, F. SCHULTMANN ${ }^{1}$, W. RASKOB ${ }^{2}$
}

\begin{abstract}
In nuclear and radiological emergency and post-accident management decisions on countermeasures and remediation strategies have to be made. Whereas in the early phase decisions on emergency actions must be made in a very short time, according to emergency plans and dose thresholds, in the recovery phase complexity is higher. But there is also more time for evaluating alternative countermeasures and remediation strategies with respect to several goals. In this paper evaluation strategies supporting the decision makers throughout all phases are presented. The applied decision support techniques range from graphical presentations of decision relevant information about the alternatives for action to multi-criteria decision making (MCDM) methods. In particular methods to cope with several types of uncertainties inherent in the different phases are presented. By the integration of these techniques, the decision support system RODOS is able to provide support for more conscious, transparent and coherent decision making.
\end{abstract}

\section{Introduction}

Both, natural and man-made disasters challenge our increasingly complex, technical and interconnected society and require an elaborate risk management. The aim of the EU 6th Framework Project EURANOS (European Approach to Nuclear and Radiological Emergency Management and Rehabilitation Strategies) (2004-2009) was, to increase coherence and effectiveness of nuclear and radiological emergency and rehabilitation management in Europe ( $c f$. Raskob et al., 2008). To this end, national emergency management organisations and research institutes worked together. While nuclear accidents refer to all incidents with substantial damage to health and/or land contamination involving fissile material, fission or a nuclear reactor, radiological events cover all other incidents related to radioactivity. Nuclear accidents differ from many other disasters by their high complexity and - fortunately - low frequency of occurrence. However, these characteristics render an adequate management difficult as approaches like contingency plans or checklists typically used in emergency management lack the flexibility needed (Geldermann et al., 2009).

1 Karlsruhe Institute of Technology (KIT, Institute for Industrial Production, Karlsruhe, Germany.

2 Karlsruhe Institute of Technology (KIT), Institut für Kern- und Energietechnik, Eggenstein-Leopoldshafen, Germany. 
In nuclear and radiological accidental management several phases - each with its own characteristics and challenges - can be distinguished. In the early phase directly after an accident (post accidental emergency phase) one of the main challenges is to get a clear picture about the contamination in order to decide upon emergency measures, e.g. evacuation or sheltering of people, distribution of stable iodine tablets. Key tasks include the monitoring of radioactivity, the simulation of atmospheric dispersion of radioactivity based on source terms and meteorological data and the calculation of geographic maps with corresponding radiological doses. Decisions have to be made quickly based on feasibility and intervention levels (Papamichail and French, 2005). In the course of the accident, the dose calculations are refined and measurement results can be combined with the results of simulations to get a clearer understanding of the situation. In the recovery phase, emergency management is complemented by post-accident management. In these phases, more time is available to make decisions. In addition, the complexity of the problem is growing: the number of management options as well as, the number and diversity of stakeholders and experts involved increases (Bertsch et al., 2006). Furthermore, the factors and criteria to be considered when selecting management options is larger, as not only factors related to health issues, but also to costs, acceptance, communication etc. gain importance. Consequently, the demands on decision support for evaluating different alternatives for action in post-accidental emergency and post-accident management differs between the phases.

The aim of this paper is to present evaluation strategies for decision support for the different phases in emergency and post-accident management in the follow-up of a nuclear or radiological accident as applied in the RODOS system (see Sect. 2). For the early phase, the paper focuses on options for visualizing the results of dose modelling and associated uncertainties. For the recovery phase, multi-attribute value theory (MAVT), a method of multi-criteria decision analysis (MCDA), is described as a method to support transparent and coherent decision making among multiple alternatives for action (measures) for complex situations with conflicting objectives (Belton and Stewart, 2002). Here, particularly the approaches in RODOS for group decision support, integration and visualisation of uncertainties as well as natural language reports of the MAVT result are emphasized. To take the dynamics of the decision process into account as well as to assess measures that are interlinked or nested and influence each other, sequential decision making is introduced.

The remainder of this paper is structured as follows: in Section 2 a brief overview of he RODOS system is provided. In Section 3 the handling and visualisation of uncertainties inherent in dose modelling are presented. Section 4 introduces the MAVT tool Web-HIPRE. In particular, its extension to account for and to visualise the impact of uncertainties, its application for group decision 

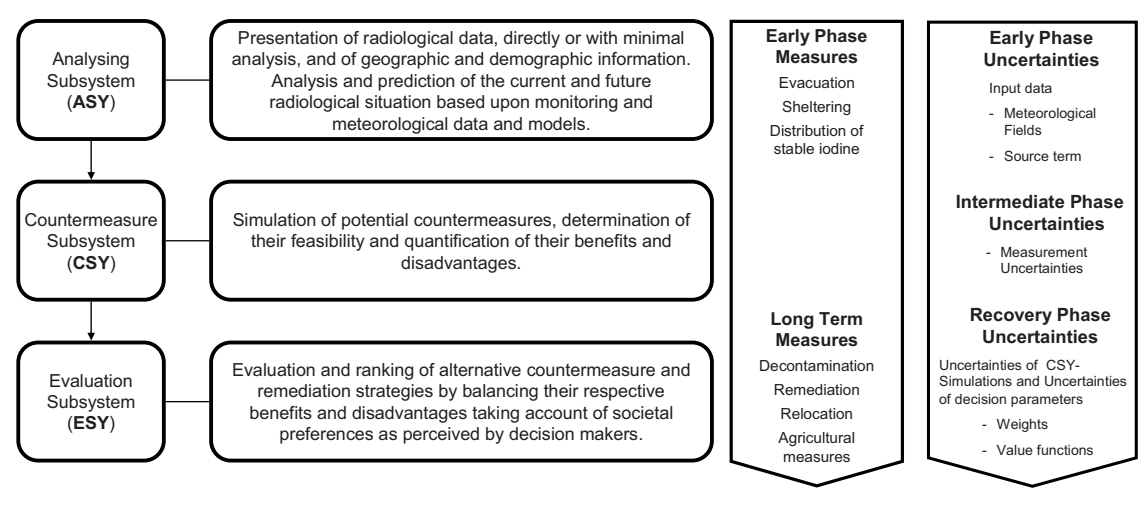

Figure 1 - The conceptual structure of RODOS.

making, and the generation of explanatory natural language reports are highlighted. Finally, Section 5 discusses sequential decision making. In Section 6 the main findings are discussed and summarised.

\section{The real time online decision support system (RODOS)}

The RODOS system was developed as a real-time online decision support system for supporting authorities and decision makers in off-site emergency and postaccident management after a nuclear or radiological accident in Europe (Ehrhardt and Weis, 2000). The level of support ranges from the provision of information in form of maps (e.g. presenting information about radiological doses) or textural reports about countermeasures to the multi-criteria assessment of measures $(c f$. Raskob et al., 2005). RODOS consists of three subsystems: the Analysing Subsystem (ASY), the Countermeasure Subsystem (CSY) and the Evaluation Subsystem (ESY) (Fig. 1). The ASY processes incoming data and predicts the location and quantity of contamination including temporal variation (Geldermann et al., 2009). To this purpose, a chain of models for atmospheric transport and dispersion, soil and plant deposition, hydrological dispersion, food chain, and radiological dose modelling is used. The dose information is used by the CSY to assess countermeasures (both, emergency actions and long-term measures) with respect to feasibility as well as quantitatively in terms of benefits and drawbacks (e.g. avoided individual and collective dose, costs) (Päsler-Sauer and Schichtel, 1997). As there is usually no measure which dominates the others in all aspects ESY has been developed to support their further evaluation and ranking by individuals or groups of decision makers. ESY uses MAVT for taking subjective 
preferences, value judgements and believes as perceived by decision makers into account. Furthermore, different kinds of uncertainties are addressed.

In nuclear and radiological emergency and post-accident management decision makers have to deal with different kinds of uncertainties. These uncertainties can e.g. be classified according to the source of the uncertainty, i.e. into uncertainties inherent in the input data (data uncertainties), uncertainties related to parameters of the applied model (parameter uncertainties) and uncertainties about the model itself (model uncertainties) ( $c f$. Morgan and Henrion, 1990; Geldermann et al., 2006; Bertsch, 2008; Raskob et al., 2009). While model uncertainties are per se difficult to handle (French and Niculae, 2005), in RODOS data uncertainties are accounted for in the ASY using ensemble modelling and visualisation techniques ( $c f$. Sect. 3). Within EURANOS, Monte-Carlo analysis was used to cope with data and parameter uncertainties in MAVT. Additionally, for the parameter uncertainties sensitivity analyses were performed ( $c f$. Sect. 4.2). In order to illustrate the impact uncertainties have on the multi-criteria decision making, visualisation techniques were developed and implemented in Web-HIPRE (Sect. 4.2).

\section{Handling and visualisation of uncertainties in dose modelling}

In the early phase of nuclear and radiological emergency management or even before the release of radioactivity into the environment, one of the key issues is to assess the spatio-temporal contamination levels and doses. This information is necessary to decide on areas where emergency measures like evacuation or distribution of stable iodine tablets must be taken. To this end, the atmospheric transport and dispersion of the radiological plume are modelled. Later on, more and more monitoring results become available. To combine all incoming information, data assimilation techniques like Kalman filtering are required ( $c f$. Gering, 2005, for a detailed presentation of Bayesian approaches used for data assimilation in the ASY). The spatio-temporal results of contamination level and dose modelling depend largely on the input data and in particular on the source term and the meteorological conditions. For the latter, the wind direction is of decisive importance. To account for uncertainties in the source term and in the wind direction, ASY performs a number of ensemble calculations based on estimates about their probability distributions ( $c f$. Gering, 2005 for details). For the source term a log-normal distribution is assumed, while for wind direction a normal distribution with $30^{\circ}$ as standard deviation is chosen (Raskob et al., 2009).

The results of the ensemble modelling must be presented in a way which can be easily understood by decision makers as time is very limited. Furthermore, a presentation which includes decision relevant information about the alternatives 


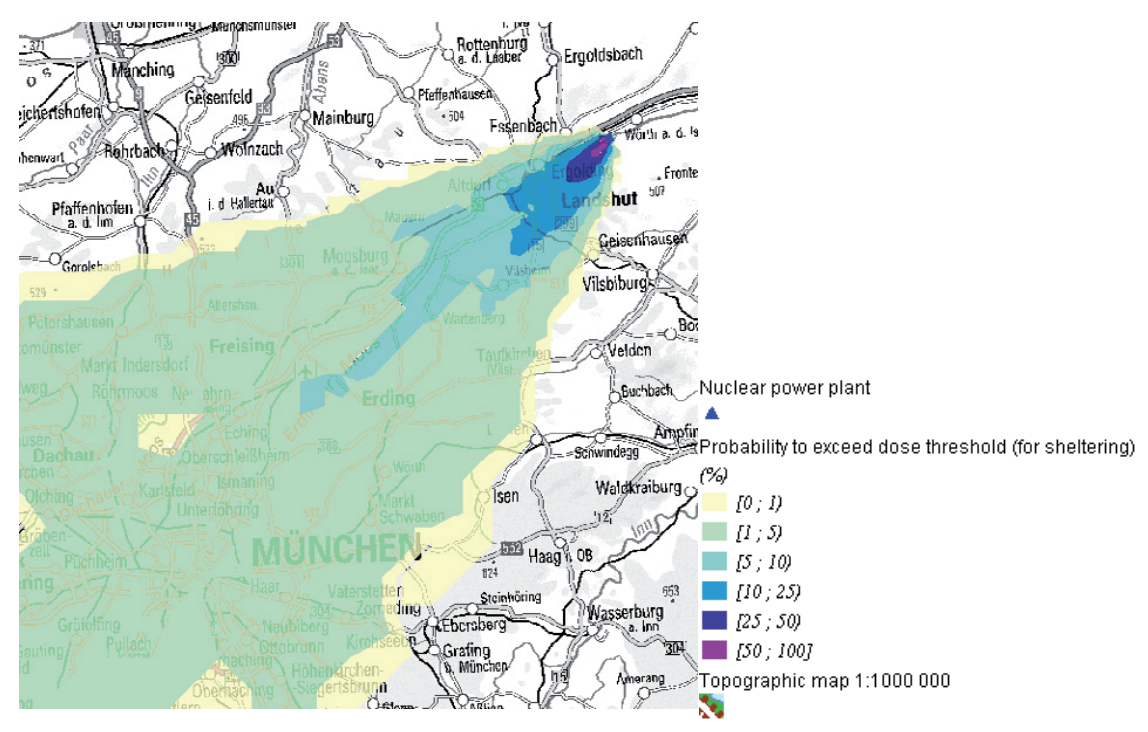

Figure 2 - Visualisation of probabilities that the intervention level for sheltering is exceeded.

for action is preferred. ASY therefore allows for displaying maps with probabilities that specific intervention levels for actions (e.g. sheltering) are exceeded ( $c f$. Fig. 2). Probability intervals are colour coded: areas where the probability of threshold exceedance is zero are not coloured. With increasing probabilities, increasingly dark and bluish colours are used. The number of intervals is kept low (in Fig. 2 just six colour levels) to avoid overwhelming the decision maker with information. Furthermore, the figures are chosen so that coding is still understandable also in grey-scale print-outs and irrespective of redgreen blindness.

\section{Multi-criteria decision analysis (MCDA) in the recovery phase}

Whenever decisions have to be made with respect to several, potentially conflicting criteria, multi-criteria decision analysis (MCDA) is considered as useful to achieve more coherent and transparent decisions (see Keeney, 1979; Stewart, 1992). However, to benefit as much as possible from MCDA, time for the elicitation of the decision makers' understanding of the problem and their preferences as well as for the interpretation and discussion of the results is needed. This limits the use of MCDA to longer term strategic emergency management MCDA, where it has proved useful, e.g. (French, 1996; Papamichail and French, 2000; Bertsch et al., 2006; Geldermann et al., 2006; Mustajoki et al., 2007). In 
RODOS, MCDA is therefore used to support decision making in post-accident management.

Two main groups of MCDA methods can be distinguished: multi-objective decision methods (MODM) and multi-attribute decision methods (MADM) ( $c f$. Belton and Stewart, 2002 for a detailed overview on MCDA). Whereas the former deal with an infinite number of alternatives (continuous solution space), the latter focus on decisions between a small number of alternatives. In emergency and postaccident management MADM is the method of choice, as in general decisions between a small number of concrete measures must be made. MADM methods can be further differentiated into methods based on multi-attribute value theory (MAVT), multi-attribute utility theory (MAUT), analytical hierarchy process (AHP) as well as outranking (Keeney and Raiffa, 1976). MAVT and MAUT have in common that the decision problem is structured by means of an attribute tree. The alternatives are ranked by the ratings of each alternative with respect to each attribute (value resp. utility) and the weights of the attributes (Mustajoki et al., 2006). While MAVT is a deterministic technique, MAUT accounts for uncertainty and the risk preferences of the decision makers which are integrated with help of probability distributions. As nuclear emergencies are rare events, these are difficult to determine in practice (Barker and Haimes, 2009). Furthermore, their use may lead to counterintuitive results (Kahneman et al., 1982; Morgan and Henrion, 1990). Therefore, MAVT has been used in the ESY.

\subsection{Multi-Attribute value theory (MAVT) and Web-HIPRE tool}

MAVT aims at ranking alternatives by combining preferences of the decision maker with attribute data. Preferences can be further differentiated into intracriteria preferences (comparisons between alternatives with respect to one criterion) and inter-criteria preferences (comparisons between criteria). In MAVT score $s_{i, a}$ of an alternative $a$ with respect to attribute $i$ is evaluated using a value function $v_{i}$ (which reflects intra-criteria preferences for attribute $i$ ) to the value $v_{i, a}$ which is then multiplied by weighting function $w_{i}$ (which reflects inter-criteria preferences) to give a partial performance score $p_{i, a}$. Weights $w_{i}$ are such that $w_{i} \geq 0, \sum_{i=1}^{n} w_{i}=1$. For $v(s)$, typically linear or exponential functions are used (e.g. Bertsch, 2008). To deduce an overall ranking, the partial performance scores are aggregated. To ensure transparency and to enhance the robustness (Stewart, 1996), partial performance scores are in ESY summed up over all $n$ criteria to the overall performance $p_{a}$ for alternative $a$ (Eq. (1)):

$$
P_{a}=\sum_{i=1}^{n} w_{i} v_{i}\left(S_{i, a}\right) \text {. }
$$




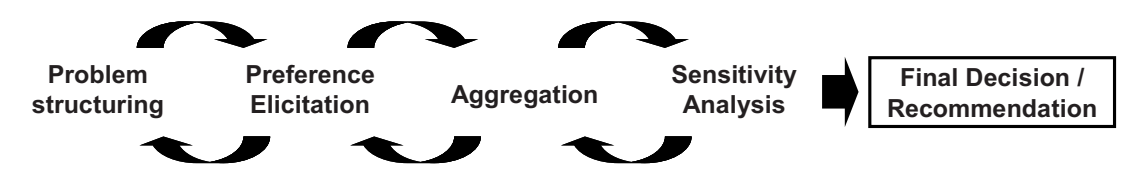

Figure 3 - Phases in multi-attribute value theory (MAVT) decision making.

Typically, MAVT comprises several phases (Fig. 3). At first, in the problem structuring phase, a so-called attribute tree is developed, i.e. the decision problem is hierarchically decomposed into criteria, sub-criteria and further on and in the end measurable attributes. Then, the preference elicitation phase serves to determine the inter- and intra-criteria preferences of the decision makers. The preferences are subsequently used to calculate partial performance scores which are aggregated in the aggregation phase to an overall performance score. Sensitivity analysis allows assessing the impact of changes of the preferences on the ranking of the alternatives. Based on both, the initial ranking and the results of the sensitivity analyses, a final decision will be made.

The java applet Web-HIPRE (www.hipre.hut.fi/) is an online software tool for multi-criteria decision analysis (MCDA) ( $c f$. Mustajoki and Hämäläinen, 2000 for an introduction) which has been adapted and integrated into the RODOS decision system within the framework of the FP5 project EVATECH (Evaluation Techniques in Nuclear Emergency Management), $c f$. also (Hämäläinen and Mustajoki, 1998; Mustajoki et al., 2007). Web-HIPRE supports a number of MCDA techniques. Decision making based on MAVT is facilitated throughout all phases: In the problem structuring phase, a GUI to develop the attribute tree can be used. Several methods for preference elicitation as well as for the subsequent aggregation of the scores are implemented. Graphical presentation of the results is supported and allows highlighting the contributions of the different attributes and (sub-)criteria to the total performance score. Furthermore, sensitivity analysis is provided ( $c f$. Sect. 4.2). Additionally, group decision making is supported ( $c f$. Sect. 4.3). In the next section extensions of Web-HIPRE in RODOS for uncertainty handling and visualisation in MAVT are described.

\subsection{Uncertainty handling and visualisation in MAVT}

In MAVT uncertainties associated with the underlying data (data uncertainties) and with the elicitation process for inter- and intra-criteria preferences (parameter uncertainties) are distinguished. Standard sensitivity analysis has the disadvantage that it can only analyse the effects of variations in one parameter at a time (OAT 

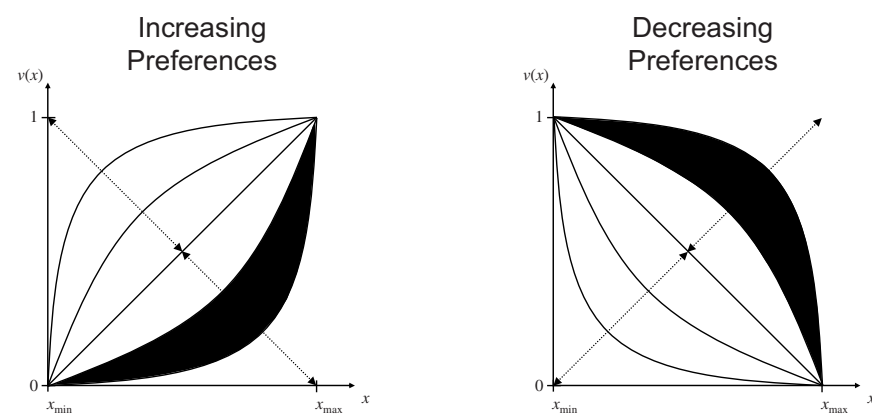

Figure 4 - Graphical support of value function sensitivity analysis (exponential value functions are determined by shape parameter $\rho_{i}$ and interval boundaries $x_{\min }$ and $\left.x_{\max }\right)$.

techniques, $c f$. French, 2003). To cope with data uncertainties and to allow multidimensional sensitivity analysis (VIC, variation in combination), MonteCarlo analysis has been applied (cf. Bertsch et al., 2006, 2008; Raskob et al., 2009). In this manner decision making becomes more conscious and a better insight in how strongly variations of uncertain parameters affect the outcomes of the decision making is gained. The use of intervals which represent the range of the decision makers' opinions instead of crisp values for inter-criteria preferences can facilitate the weight elicitation process ( $c f$. Bertsch and Geldermann, 2008). Whereas the assignment of intervals for inter-criteria preferences is straightforward, uncertainties with respect to the value functions (intra-criteria preferences) are more difficult to account for. Therefore, value function sensitivity analysis is graphically supported (Fig. 4) (cf. Bertsch, 2008; Hiete et al., 2009).

One of the key issues when coping with uncertainties is the communication of their effects on the results. In the composite bar chart visualisation of the alternatives' (mean) overall performance scores in Web-HIPRE, additional bars for 5\% and $95 \%$ quantiles were added ( $c f$. Fig. 5). This shows how uncertainties affect overall performance scores and provides some insight how the uncertainties affect criteria-specific scores of the alternatives. To some extent $5 \%$ and $95 \%$ quantile bars can be regarded as "best" and "worst case" results for the alternatives. Figure 5 clearly shows that some alternatives (e.g. "AddS+Proc") are rather robust against the expected uncertainties of underlying data and preferences - reflected in a similar height of bars - and that changes in the overall performance score are mainly due to changes in the contribution of the criterion "impact". 


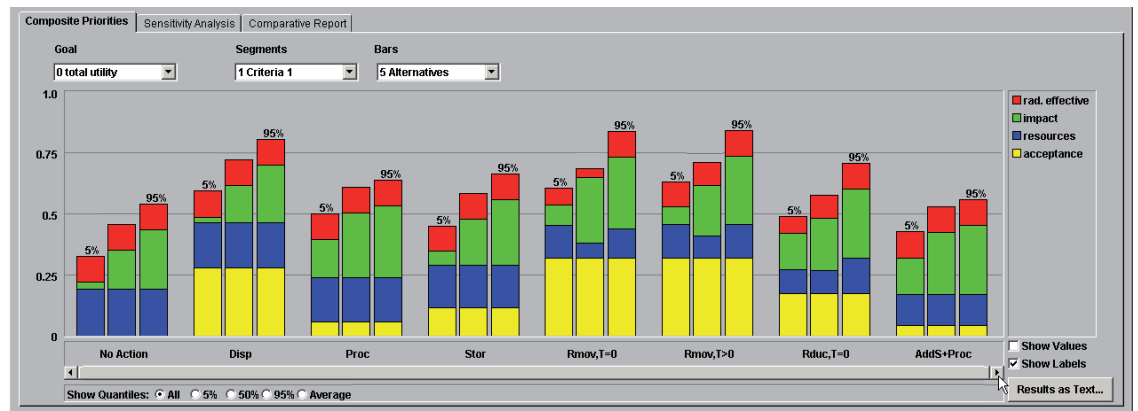

Figure 5 - Visualisation of the effects of data and parameters uncertainties on overall performance score (heights of the bars) and on the four main criteria contributing to overall performance score of eight alternatives for action (main criteria: radiological effectiveness, impact, resources used, and acceptance by stakeholders; alternatives (examples), e.g. "Disp." for milk disposal; “Rmov, $T=0$ " and "Rmov, $T>0$ " for moving cows in the stable before resp. two days after the release and feeding with uncontaminated feed, "AddS+Proc" for adding sorbents to the feed and milk processing).

To allow examination of the sensitivity of the results in the worst, mean and best cases against parameter variations, sensitivity analyses can be performed and visualised for all three percentiles (not shown). In addition, a case wise analysis of the scores enables the user to determine which alternatives achieve how frequently the highest performance. For the example shown in Figure 5, in 77\% of all cases, the alternative "Rmov, $T=0$ " gets the highest overall performance score followed by the alternative "Disp." in $23 \%$ of the cases. Overall, this allows the decision maker to get insights about the effects that both data and parameter exert on the MAVT results and to identify the parameters and data with the highest influence on the ranking which therefore need special attention.

\subsection{Group decision making}

In post-accident management experts with different backgrounds, competences and knowledge have to work together in order to identify the best actions. Via its structured and transparent approach MAVT is an ideal technique to support decision making in these heterogeneous groups (Stewart, 1992; French and Geldermann, 2005). Developing the attribute tree in a group and discussing preferences can be effective in developing more conscious decisions and integrating different views. One prerequisite is, however, that the decision making process is transparent and understandable for the decision makers. Here MAVT is advantageous, in particular if the weighted sum aggregation method is applied. 
Web-HIPRE supports per se group decision making but only as a weighted sum of the individual decision makers' priorities (Mustajoki et al., 2004; Bertsch and Geldermann, 2008). As a web-based tool, group members can access it, although they may be geographically dispersed.

Especially sensitivity analysis can be useful to get to a consensus for the preferences, as it facilitates assessing the impact of a change in preferences on the ranking of alternatives. This can avoid lengthy and controversial group discussions. Furthermore, the newly integrated Monte Carlo analysis proved to be very useful for group decision making problems (Bertsch, 2008). Discussions on both data and preferences can be sped up if $e . g$. ranges representing the variety of opinions of the group instead of crisp values can be used as input to MCDA. In particular, if the ranking of the alternatives does not change within the proposed range, controversial discussions on values can be avoided. Coming from the effects-side can thus be beneficial for group decision making.

\subsection{Natural lanuage reports}

In Web-HIPRE natural language reports for explaining the results of the decision making are integrated (Geldermann et al., 2009). Two reports explaining the results using in English language are generated:

- The comparative report compares two alternatives with arguments in favour as well as for the disadvantage of each alternative and explains the factors differentiating between both alternatives.

- The sensitivity analysis report interprets the sensitivity graph by explaining the effects a of variation in an attribute weight and provides information about robustness of the results.

For report generation both natural language techniques (e.g. Reiter and Dale, 2000) and statistical methods (e.g. Klein, 1994) are used (cf. Papamichail and French, 2003; Geldermann et al., 2009 for a more detailed description). Providing natural language reports in addition to graphical output has several advantages in MCDA: first, a decision maker who is not familiar with the graphical representations is provided with an easily understandable interpretation. This fosters not only the understanding of the novice but also of the experienced decision maker. Second, subjective interpretations of the results and cognitive biases are reduced. Third, this approach facilitates discussion as it guarantees that all decision makers share a common interpretation of the results. Another advantage is that these reports can be easily communicated to outsiders or the public. Overall these reports can increase confidence in the decision making and acceptance in the results ( $c f$. e.g. Papamichail and French, 2003 and literature cited therein). 


\section{Sequential decision making}

Although in emergency management decision makers are confronted with a series of nested decisions that are related to each other (French and Ríos-Insua, 2000), MCDA is usually limited to decisions made at one point in time. Contrarily, Sequential Decision Making (SDM) provides a framework to facilitate decision making in situations where a series of decisions needs to be made. To facilitate the problem structuring in these situations, two methods for graphical representation are available: influence diagrams and decision trees (Clemen and Reilly, 1999).

One way to deal with these sequential decisions is to introduce flexibility as an additional criterion in MCDA (Benjaafar et al., 1995). This approach allows for integrating the number of feasible future decision options into the reasoning process. But it is not clear, that flexibility is a value in itself: Assume e.g., that a decision alternative $A$ requires the application of $A^{\prime}$ in a later phase, whereas an alternative B allows for applying both A' and B'. Obviously, B is more flexible than A. However, if the sequence A-A' is evaluated better than both B-A' and BB', then option A should be recommended - although it is less flexible. Therefore, a more exhaustive analysis of sequential decision making is needed.

In the context of EURANOS, an analysis of pairs of countermeasures throughout all phases of emergency and post-accident management, in inhabited as well as in agricultural areas has been performed ( $c f$. www.euranos.fzk.de/ for generic handbooks on countermeasures). It has been shown that - beyond the distinction of emergency and recovery phase countermeasures - for some measures the time of their application as well as the order in which they are applied can have an impact on the results. For example, while it is recommended to shelter the population before starting a possibly necessary evacuation (Brown et al., 2007), applying the measures in reversed order (first evacuate, then shelter) is not appropriate. Furthermore, while some measures do have an effect over a longer period of time (e.g. roof replacement, roof brushing, roof cleaning with hot water which can be effective up to 10 years after the deposition) others are more related to time or weather constraints (e.g. snow removal) (cf. Brown et al., 2007). While these remarks seem to be straightforward, they show that it is important to take interdependencies into account. SDM founded on the pairwise interdependency analysis facilitates taking into account the order and timing of each step in planning and evaluating mitigation strategy.

\section{Conclusions}

In nuclear and radiological emergency and post-accident management several phases can be distinguished. In the early phase time is critical. Therefore, fast 
determination and assessment of the contamination as a prerequisite for decision making on emergency countermeasures is of highest priority. Contrarily, in the post-accident phase when it comes to decide on remediation measures more time is available for decision making. However, now the number of management options as well as the number and diversity of stakeholders and experts involved is growing, and thus, the complexity of the decision problem.

The decision support system RODOS for off-site emergency management provides support for evaluation of countermeasures adapted to the different phases following a nuclear or radiological emergency. In the early phase the ASY of RODOS provides decision relevant information about the potential contamination of the environment and doses to the public. A key issue is the presentation of maps showing radiological threshold exceedances associated with intervention levels (e.g. trigger for countermeasures). To account for the uncertainties in radiological doses colour coding for the exceedance probabilities has been proposed.

In the recovery phase more time to decide upon the potential countermeasures simulated by RODOS' CSY is available. However, the problem is also increasingly complex. Multi-criteria decision analysis (MCDA) and in particular multi-attribute value theory (MAVT) has been used to achieve more coherent and transparent decisions in such situations with several, potentially conflicting objectives. Web-HIPRE, an online software tool for MCDA, was integrated in RODOS' ESY and adapted to the particular needs in post-accident management. Monte-Carlo techniques and sensitivity analyses were integrated to cope with data and parameter uncertainties. To ensure comprehensibility and clarity, adapted visualisation techniques were developed. Interpretation of Web-HIPRE results is further facilitated by natural language reports. Altogether, the integrated uncertainty handling approaches also facilitates decision making in groups. As decisions on single remediation measures are usually heavily dependent on each other, the countermeasures simulated by the CSY have been analysed pair by pair for impacts of their order and time of application on the results. However, the recommendations made based on this first analysis need more refinement to cope with the multiplicity of interlinkages between the countermeasures leaving large space for research on sequential decision making.

To conclude, the evaluation strategies developed and implemented in RODOS provide the user with a variety of tools and possibilities to improve decision making in nuclear and radiological emergency and post-accident management, in particular when uncertainties are prevailing. For highly uncertain and fundamentally varying situations, however, combining MCDA with scenariobased reasoning (SBR) looks fruitful (Comes et al., 2009). The way scenarios deal with uncertainty is appealing to users, as scenarios decompose complexity by 
presenting several plausible developments of the world (Chermack, 2004). The combination of MCDA \& SBR supports decision makers in selecting a strategy whose performance is robust to a range of scenarios that encompass all plausible and relevant futures (Comes et al., 2009). The application of this novel technique in nuclear and radiological emergency and post-accident management is another field of ongoing research.

Acknowledgment. This work has received partial financial support from the European Commission Sixth Framework Programme (Nuclear Fission/Radiation Protection) under the EURANOS integrated project: European approach to nuclear and radiological emergency management and rehabilitation strategies (Contract No: FI6R-CT-2004-508843).

\section{REFERENCES}

Barker K., Haimes Y.Y. (2009) Assessing uncertainty in extreme events: Applications to risk-based decision making in interdependent infrastructure sectors. Reliab. Eng. Syst. Saf. 94, 819-829.

Belton V., Stewart T. (2002) Multiple Criteria Decision Analysis. An Integrated Approach. Kluwer Academic Publishers., Boston.

Benjaafar S., Morin T.L., Talavage J.J. (1995) The strategic value of flexibility in sequential decision making, Eur. J. Oper. Res. 82, 438-457.

Bertsch V. (2008) Uncertainty handling in Multi Attribute Decision Support for Industrial Risk Management. Dissertation, Universität Karlsruhe (TH), Karlsruhe University Press, available online at: http://digbib.ubka.uni-karlsruhe.de/volltexte/1000007378.

Bertsch V., Geldermann J. (2008) Preference elicitation and sensitivity analysis in multicriteria group decision support for industrial risk and emergency management, IJEM 5, 7-24.

Bertsch V., Geldermann J., Rentz O., Raskob W. (2006) Multi-Criteria Decision Support and Stakeholder Involvement in Emergency Management, IJEM 3, 114-130.

Brown J., Mortimer K., Andersson K., Duranova T., Mrskova A., Hänninen R., Ikäheimonen T., Kirchner G., Bertsch V., Gallay N., Hammond D., Kwakman P. (2007) Generic Handbook for Assisting in the Management of Contaminated Inhabited Areas in Europe Following a Radiological Emergency. Report within the EURANOS project. European Commission (EC).

Chermack T.J. (2004) Improving decision-making with scenario planning, Futures 36, 295-309.

Clemen R., Reilly T. (1999) Making Hard Decisions with DecisionTools. Pacific Grove, CA, Duxbury.

Comes T., Hiete M., Wijngaards N., Kempen M. (2009) Integrating Scenario-Based Reasoning into Multi-Criteria Decision Analysis. In: Proceedings of the 6th International Conference on Information Systems for Crisis Response and Management (ISCRAM 2009), May 10-13, 2009, Gothenburg, Sweden.

Ehrhardt J., Weis A. (2000) RODOS: Decision Support System for Off-site Nuclear Emergency Management in Europe. European Commission, Brussels, Report EUR 19144.

French S. (1996) Multi-attribute decision support in the event of a nuclear accident, J. Multi-Criteria Decis. Anal. 5, 39-57.

French S. (2003) Modelling, making inferences and making decisions: The roles of sensitivity analysis, TOP 11, 229-251.

French S., Geldermann J. (2005) The varied contexts of environmental decision problems and their implications for decision support, Environmental Science \& Policy 8, 378-391. 


\section{H. HIETE et al.}

French S., Niculae C. (2005) Believe in the Model: Mishandle the Emergency. JHSEM 2, Available at: http://www.bepress.com/jhsem/vol2/iss1/2, accessed November 13, 2009.

French S., Ríos-Insua D. (2000) Statistical Decision Theory. London: Edward Arnold.

Geldermann J., Treitz M., Bertsch V., Rentz O. (2005) Moderated Decision Support and Countermeasure Planning for off-site Emergency Management. In: Energy and Environment: Modeling and Analysis. (Loulou R., Waaub J.-P., Zaccour G., Eds.) Kluwer Academic Publishers.

Geldermann J., Bertsch V., Rentz O. (2006) Multi-Criteria Decision Support and Uncertainty Handling, Propagation and Visualisation for Emergency and Remediation Management. In: (H.-D. Haasis, H. Kopfer, J. Schönberger, Eds.), Operations Research Proceedings 2005, pp. 755-760, Springer, Available at: http://dx.doi.org/10.1007/3-540-32539-5_118.

Geldermann J., Bertsch V., Treitz M., French S., Papamichail K.N., Hämäläinen R.P. (2009) Multicriteria Decision Support and Evaluation of Strategies for Nuclear Remediation Management. OMEGA, The International Journal of Management Science 37, 238-251.

Gering F. (2005) Data assimilation methods for improving the prognoses of Radionuclide Deposition from radioecological models with measurements, $\mathrm{PhD}$ thesis, Leopold-Franzens-University Innsbruck, Austria.

Hämäläinen R.P., Mustajoki J. (1998) Web-HIPRE Java Applet for Value Tree and AHP Analysis. Computer software, Systems Analysis Laboratory, Helsinki University of Technology.

Hiete M., Ludwig J., Merz M., Bertsch V. (2009) Ökodesign von Gebäuden - Visualisierungstechniken für Mehrzielentscheidungsprobleme. In: Methods of Multicriteria Decision Theory and Applications, (Heyde F., Löhne A., Tammer Ch., Eds.) pp. 57-78, Shaker Verlag Aachen.

Kahneman D., Slovic P., Tversky A. (1982) Judgment under Uncertainty: Heuristics and biases. Cambridge: Cambridge University Press.

Keeney R.L., Raiffa H., Rajala D.W. (1979) Decisions with Multiple Objectives: Preferences and Value Trade-Offs, IEEE Transactions on Systems, Man and Cybernetics 9, 403.

Keeney R.L., Raiffa H. (1976) Decisions with Multiple Objectives. New York: John Wiley \& Sons.

Klein D.A. (1994) Decision-analytic intelligent systems: automated explanation and knowledge acquisition. Lawrence Erlbaum Associates, New Jersey.

Morgan M.G., Henrion M. (1990) Uncertainty: A Guide to Dealing with Uncertainty in Quantitative Risk and Policy Analysis. Cambridge University Press, Cambridge.

Mustajoki J., Hämäläinen R.P. (2000) Web-HIPRE: global decision support by value tree and AHP analysis, INFOR 38, 208-220.

Mustajoki J., Hämäläinen R.P., Lindstedt M.R.K. (2006) Using intervals for global sensitivity and worst-case analyses in multiattribute value trees, Eur. J. Operat. Res. 174, 278-292.

Mustajoki J., Hämäläinen R.P., Marttunen M. (2004) Participatory multicriteria decision analysis with Web-HIPRE: a case of lake regulation policy, Environ. Modell. Softw. 19, 537-547.

Mustajoki J., Hämäläinen R.P., Sinkko K. (2007) Interactive computer support in decision conferencing: Two cases on off-site nuclear emergency management, Decis. Support Syst. 42, 2247-2260.

Papamichail K.N., French S. (2000) Decision support in nuclear emergencies, J. Hazard. Mater. 71, 321-342.

Papamichail K.N., French S. (2003) Explaining and justifying the advice of a decision support system: a natural language generation approach, Exp. Syst. Appl. 24, 35-48.

Papamichail K.N., French S. (2005) Design and evaluation of an intelligent decision support system for nuclear emergencies, Decis. Support Syst. 41, 84-111.

Päsler-Sauer J., Schichtel T. (1997) The Simulation of Early Emergency Actions in RODOS, Radiat. Prot. Dosim. 73, 71-74. 


\section{EVALUATION STRATEGIES FOR NUCLEAR AND RADIOLOGICAL EMERGENCY}

Raskob W., Bertsch V., Geldermann J., Baig S., Gering F. (2005) Demands to and experience with the Decision Support System RODOS for off-site emergency management in the decision making process in Germany. In: Proceedings of the Second International Conference on Information Systems for Crisis Response and Management, (Van de Walle B., Carlé B., Eds.), Brussels, Belgium, pp. 269-278.

Raskob W., Gering F., Lochard J., Nisbet A., Starostova V., Tomic B. (2008) RODOS: Overview and main achievements of the EURANOS project: European Approach to Nuclear and Radiological Emergency Management and Rehabilitation Strategies. In: Proceedings of the International Conference on Radioecology and Environmental Radioactivity, June 15-20, Bergen, Norway.

Raskob W., Gering F., Bertsch V. (2009) Approaches to visualisation of uncertainties to decision makers in an operational Decision Support System. In: Proceedings of the 6th International Conference on Information Systems for Crisis Response and Management (ISCRAM 2009), May 10-13, Gothenburg, Sweden.

Reiter E., Dale R. (2000) Building applied natural language generation systems. Cambridge University Press, Cambridge, UK.

Stewart T.J. (1996) Robustness of Additive Value Function Methods in MCDM, J. Multi-Criteria Decis. Anal. 5, 301-309.

Stewart T.J. (1992) A critical survey on the status of multiple criteria decision making theory and practice, Omega 20, 569-586. 